\title{
On Source Space Resolution in EEG Brain Imaging for Motor Imagery
}

Radu Ranta, Steven Le Cam, Gundars Bergmanis-Korats, Sébastien Rimbert, Laurent Bougrain

\section{- To cite this version:}

Radu Ranta, Steven Le Cam, Gundars Bergmanis-Korats, Sébastien Rimbert, Laurent Bougrain. On Source Space Resolution in EEG Brain Imaging for Motor Imagery. International IEEE EMBS Conference on Neural Engineering, Mar 2019, San Francisco, France. <hal-01985178>

\section{HAL Id: hal-01985178 \\ https://hal.inria.fr/hal-01985178}

Submitted on 17 Jan 2019

HAL is a multi-disciplinary open access archive for the deposit and dissemination of scientific research documents, whether they are published or not. The documents may come from teaching and research institutions in France or abroad, or from public or private research centers.
L'archive ouverte pluridisciplinaire HAL, est destinée au dépôt et à la diffusion de documents scientifiques de niveau recherche, publiés ou non, émanant des établissements d'enseignement et de recherche français ou étrangers, des laboratoires publics ou privés. 


\title{
On Source Space Resolution in EEG Brain Imaging for Motor Imagery
}

\author{
Radu Ranta ${ }^{1}$, Steven Le Cam ${ }^{1}$, Gundars Bergmanis-Korats ${ }^{2}$, Sébastien Rimbert ${ }^{3}$ and Laurent Bougrain ${ }^{3}$
}

\begin{abstract}
Brain source localization accuracy is known to be dependent on the EEG sensor placement over the head surface. In Brain-Computer Interfaces (BCI), according to the paradigm used, Motor Imagery (MI) and Steady-State Visual Evoked Potential (SSVEP) in particular, electrodes are not well distributed over the head, and their number is not standardized as in classical clinical applications. We propose in this paper a method for quantifying the expected quality of source localization with respect of the sensor placement, known as EEG montage. Our method, based on a subspace correlation metric, can be used to assess which brain sources can be distinguished (as they generate sufficiently different potentials on the electrodes), and also to identify regions/volumes in which precise source localization is impossible (i.e. all sources inside those regions could generate similar electrode potentials). In particular, for a MI dedicated montage, we show that source localization is less precise than for standard montages, although the local density of electrodes over the areas of interest is higher.
\end{abstract}

\section{INTRODUCTION}

Source localization is seldom used in EEG-based BrainComputer Interface (BCI) applications (with some notable exceptions, e.g. [1], [2], [3], [4]), for several good reasons. Among them, one of the most important is the electrode placement. Indeed, properly addressing the source localization problem implies processing signals coming from surface EEG electrodes, in a sufficient number and well distributed over the head surface [5]. On the contrary, for practical reasons mostly (supposed areas of interest, installation time, comfort of the subject), most of end-user BCI applications use surface electrodes in a small number placed over specific brain regions (motor/sensitive areas for motor imagery for example).

The aim of this work is to propose a systematic and rigorous approach for exploring the feasibility of source localization from BCI-like electrode setups. Our approach is based on a subspace correlation measure exploited as an euclidean distance and fed into a clustering algorithm. The result is a parcelation of the brain volume in distinguishable regions, depending on the electrode placement. Within each region, the sources are virtually impossible to distinguish, meaning that every such region could be approximated by a unique equivalent source. The proposed approach yields thus

\footnotetext{
*This work was supported by CNRS PEPS 2018 project Move Your Mind

${ }^{1}$ R. Ranta and S. Le Cam are with Université de Lorraine, CNRS, CRAN, F-54000 Nancy, France, radu.ranta@univ-lorraine.fr, steven.le-cam@univ-lorraine.fr

${ }^{2}$ G. Bergmanis-Korats is with Ventspils University of Applied Sciences, Ventspils Smart Technology Research Centre, Ventspils, Latvia

${ }^{3}$ S. Rimbert and L. Bougrain are with the Université de Lorraine, CNRS, Inria, LORIA, F-54000 Nancy, France sebastien.rimberteinria. fr, laurent.bougrain@loria.fr
}

a coarse but irregular discretization of the brain volume, with well defined (small) and badly defined (big) regions whose repartition depends on the electrode setup. It is noteworthy that other authors have proposed a coarse discretization. For example, in [6], the authors propose to subsample the brain volume based on physiologically/anatomically defined brain atlases.

\section{SOURCE LOCALIZATION BASICS}

Electric brain source localization consists in determining the positions, orientations and strength of neural sources using the electrical potential measurements, usually acquired by scalp EEG electrodes. Formally, this can be stated as an inverse problem: knowing some measurements and a model linking the sources to the measurements (here a propagation model), one wants to estimate the sources that have generated these measurements. There are a set of common assumptions for all localization methods:

- at each time instant $t$, a source can be modeled as a current dipole, and thus characterized by 6 parameters per source: the position $\mathbf{r}_{n}=\left(x_{n}, y_{n}, z_{n}\right)$, $(3 \times 1)$, and the dipolar moment vector $\mathbf{j}_{n}(t)=$ $\left(j_{n, x}(t), j_{n, y}(t), j_{n, z}(t)\right),(3 \times 1)$, where the three components are the amplitudes of the projections along the $x, y, z$ axis;

- the propagation coefficients $\mathbf{k}_{m, n},(3 \times 1)$, between the $n$-th source (at position $\mathbf{r}_{n}$ ) and the $m$-th electrode do not vary in time and they do not depend on the timefrequency characteristics of the sources.

Basically, these assumptions come both from neuroanatomical considerations (alignment of the pyramidal neurons in the cortex for the source model) and Maxwell equations for the electromagnetic fields at low frequencies (for the propagation model) (see e.g. [7] for more details on the modeling).

The previous assumptions allow to model at each time $t$ the potentials on the head surface as a linear combination of dipolar sources:

$$
\mathbf{V}=\mathbf{K J}
$$

where $\mathbf{V}(M \times 1)$ are the potentials on the $M$ electrodes, $\mathbf{K}$ $(M \times 3 N)$ is the so-called lead-field matrix and $\mathbf{J},(3 N \times 1)$ are the (elementary) dipolar moments.

The lead-field matrix $\mathbf{K}$ can be obtained using analytic or numerical approaches. For example, if the head is approximated by one or several concentric spheres (standing e.g. for brain, skull, scalp), one can derive analytic solutions for every point on the surface of the spheres. More elaborated models include a realistic model of the head geometry, extracted from imagery modalities (CT scan, MRI). These 
numerical models can be limited to surfaces (e.g. for brain, skull, scalp), in which case they are known as BEM (Boundary Elements Methods) or they can discretize the volumes also (including separate discretization of gray/white matter if needed). Among these last solutions, the most used are the FEM (Finite Elements Methods).

Apart from the geometry, one needs to make assumptions on the electrical properties of the tissues in these different volumes (or finite elements). The widely accepted hypothesis is that the tissues are purely resistive, i.e. they are characterized by their conductivities $\sigma$ (which in principle can be different by spatial direction also).

To conclude, the lead-field matrix, which is at the basis of all source-localization procedures, is computed as a function of the medium characteristics (geometry and conductivity) and the positions of the considered possible sources and the electrodes. Usually, the $N$ possible source positions are defined as a grid inside the brain volume, with a resolution depending on the accuracy of our model, while the electrodes are in far smaller number $M \ll N$. $\mathbf{K}$ matrix is thus not invertible, and different ways of finding the sources from (1) have been proposed in the literature over the last 30 years (see e.g. [8]).

\section{SUbSPACE CORRELATION METRIC}

A lead field matrix $\mathbf{K}(M \times 3 N)$ can thus be seen as a collection of $N$ groups of 3 vectors in $\mathbb{R}^{M}$, each group related to a source position. Formally speaking, every group defines a $3 D$ subspace of $\mathbb{R}^{M}$. This means that any current density vector $\mathbf{j}_{n}$ associated to the position $n \in\{1 \ldots N\}$ (i.e. pointing in any direction and having any amplitude) lies in the $n$-th subspace, as it can be written as a linear combination between the 3 vectors defining the respective subspace.

The question one can ask is: are there two $3 D$ subspaces $\mathcal{S}_{i}$ and $\mathcal{S}_{j}$, and, within each subspace, two current vectors $\mathbf{j}_{i}$ and $\mathbf{j}_{j}$ having identical projections on the sensors? In other words, is it possible to obtain the same scalp potentials from sources lying at two different positions (but with potentially different orientations and amplitudes)? The answer to this question is given by the dimension of the common subspace between $\mathcal{S}_{i}$ and $\mathcal{S}_{j}$.

Consider two groups of 3 vectors forming two matrices $\mathbf{K}_{i}$ and $\mathbf{K}_{j}(M \times 3)$. These matrices, assumed linearly independent, define the $3 \mathrm{D}$ subspaces $\mathcal{S}_{i}$ and $\mathcal{S}_{j}$ associated to the positions $n_{i}$ and $n_{j}$. A basis for each subspace can be obtained for example by whitening: after computing the product $\boldsymbol{\Phi}_{i}=\mathbf{K}_{i}^{T} \mathbf{K}_{i}$, the basis vectors $\mathbf{Z}_{i}$ are obtained as follows:

$$
\mathbf{Z}_{i}=\mathbf{K}_{i} \mathbf{U}_{i} \mathbf{D}_{i}^{-1 / 2}
$$

with $\mathbf{D}_{i}$ and $\mathbf{U}_{i}$ respectively the diagonal matrix of eigenvalues and the eigenvector matrix of the matrix $\boldsymbol{\Phi}_{i}$. Strictly the same development yields a basis $\mathbf{Z}_{j}$ of $\mathcal{S}_{j}$. Of course, these solutions are not unique, any orthogonal (rotation) matrix will yield alternative basis vectors for the same subspaces. The question of the common subspace between $\mathcal{S}_{i}$ and $\mathcal{S}_{j}$ becomes then: are there some particular rotations that we can apply to $\mathbf{Z}_{i}$ and $\mathbf{Z}_{j}$ such as they become aligned, i.e. their 3 basis vectors become identical?

An simple solution for finding two rotation matrices $\mathbf{V}$ and $\mathbf{W}(3 \times 3)$ that will align the subspaces spanned by $\mathbf{Z}_{i}$ and $\mathbf{Z}_{j}$ is given in Golub and Loan [9, p. 603-605]. It starts by computing the singular value decomposition of the $3 \times 3$ matrix $\mathbf{Z}_{i}^{T} \mathbf{Z}_{j}$ :

$$
\mathbf{Z}_{i}^{T} \mathbf{Z}_{j}=\mathbf{V} \boldsymbol{\Sigma} \mathbf{W}^{T}
$$

Let

$$
\begin{aligned}
\mathbf{Y}_{i} & =\mathbf{Z}_{i} \mathbf{V} \\
\mathbf{Y}_{j} & =\mathbf{Z}_{j} \mathbf{W}
\end{aligned}
$$

be the rotated versions of the $\mathbf{Z}_{i}$ and $\mathbf{Z}_{j}$ basis, thus themselves also basis of $\mathcal{S}_{i}$ and $\mathcal{S}_{j}$. It is easy to see that

$$
\mathbf{Y}_{i}^{T} \mathbf{Y}_{j}=\boldsymbol{\Sigma}
$$

meaning that a vector $k$ in $\mathbf{Y}_{i}$ is orthogonal to all vectors $l \neq k$ from $\mathbf{Y}_{j}$ and that the cosine of the angle between vectors having the same index $k$ in both $\mathbf{Y}_{i}$ and $\mathbf{Y}_{j}$ basis is given by the $k$-th singular value $\sigma_{k}$. In particular, if $\sigma_{k}=1$, the $k$-th vectors from the two basis are aligned. If the three basis vectors are aligned (i.e. $\Sigma$ is the identity matrix), then the two subspaces are aligned.

In principle, with a non-singular lead-field matrix, this is not possible. Indeed, if two subspaces are completely aligned, it means that the columns of the lead-field corresponding to one of the subspaces can be written as a linear combination of the columns corresponding to the other subspace. But, on the other hand, it is fairly possible and quite current for two subspaces to be "approximately" aligned: if the grid of source positions is dense and the sensors are far, it is quite naturally that the potentials on the sensors will be very similar, approximately the same.

We can define thus a "distance" between two subspaces in order to quantify how similar they are. We propose the smallest singular value of $\Sigma$ in (5). This can be seen as the euclidean distance between the third basis vectors from $\mathbf{Y}_{i}$ and $\mathbf{Y}_{j}$. Indeed, if we note these vectors as $\mathbf{y}_{i, 3}$ and $\mathbf{y}_{j, 3}$, simple geometrical considerations show that the squared distance writes:

$$
\begin{aligned}
d_{i, j}^{2} & =\left\|\mathbf{y}_{i, 3}-\mathbf{y}_{j, 3}\right\|_{2}^{2} \\
& =\left\|\mathbf{y}_{i, 3}\right\|_{2}^{2}+\left\|\mathbf{y}_{j, 3}\right\|_{2}^{2}-2\left\|\mathbf{y}_{i, 3}\right\|_{2}\left\|\mathbf{y}_{j, 3}\right\|_{2} \cos \phi_{i j, 3} \\
& =2\left(1-\cos \phi_{i j, 3}\right)
\end{aligned}
$$

where $\phi_{i j, 3}$ is the angle between the vectors $\mathbf{y}_{i, 3}$ and $\mathbf{y}_{j, 3}$, both having unit norm by construction.

Expression (6) defines thus an euclidean distance between the subspaces $\mathcal{S}_{i}$ and $\mathcal{S}_{j}$ related to locations $n_{i}$ and $n_{j}$. A small distance will imply that there exists two dipoles, situated at these two positions, that project very similarly on the electrodes. In other words, they are potentially indistinguishable, especially in the presence of noise. 


\section{Clustering}

Given a distance between any pair of source positions in the head, one can use it to construct clusters of source positions: close positions (in terms of distance (6)) are impossible to identify by any inverse problem technique, because they project similarly onto the sensors. Consequently, one can take for example the centroid of every cluster as a representative source for the whole cluster, and thus potentially subsample the lead-field matrix. This reduced lead-field matrix can significantly speed up computations and, moreover, it might indicate quantitatively the precision limits of source localization algorithms depending on the positions inside the head volume.

We propose here a completely unsupervised clustering procedure, based on agglomerative hierarchical clustering (AHC) [10]. In brief, AHC starts by initializing each cluster to a point. So at the beginning, we have as many clusters as points. Then an iterative procedure i) selects the two closest clusters and ii) agglomerates them into a new cluster until a stopping criterion is reached (pre-defined number of clusters, maximum distance between the closest clusters, ...). The first step depends on a distance criterion such as the minimum distance between any points of two different clusters. The second step, called linkage, depends thus on the cluster merging criterion. Several linkage procedures have been proposed [10]. In our application, we aim to merge two clusters only if the projection of the dipoles within those two clusters onto the sensors (i.e. the forward problem) risk to be impossible to distinguish. In particular, the two most different dipoles in the two clusters need to be similar enough. We choose then the distance between two clusters and thus the resulting linkage procedure as the complete linkage (also known as farthest neighbour).

A very important observation is that the subspaces involved in this argumentation are groups of lead-field vectors. Consequently, they depend on the used model and, most important, on the positions of the sensors. The subspaces associated to two positions, and thus their angles and thus distance (6), depend on the employed sensors. If their number is high or low, or if their positions are regularly distributed over the head or concentrated onto specific areas can dramatically change the clustering and thus the spatial sampling or the "resolution" of the obtained reduced lead-field.

The AHC procedures continues iteratively until all the points are merged together in a unique cluster. A common graphical representation is the dendrogram, which is a binary tree-like structure associating at each node two clusters in a bigger cluster (see fig. 1 for an example on our data). If our aim is to cluster the points in similar regions, we have to cut the dendrogram in order to separate the different branches of the binary tree. Different procedures exist but, again guided by our application, we choose to fix a distance threshold: if two clusters are farther away than that threshold (meaning that the maximum distance between any two points in the two clusters is bigger than the threshold), then we consider them as separate regions. The threshold we propose

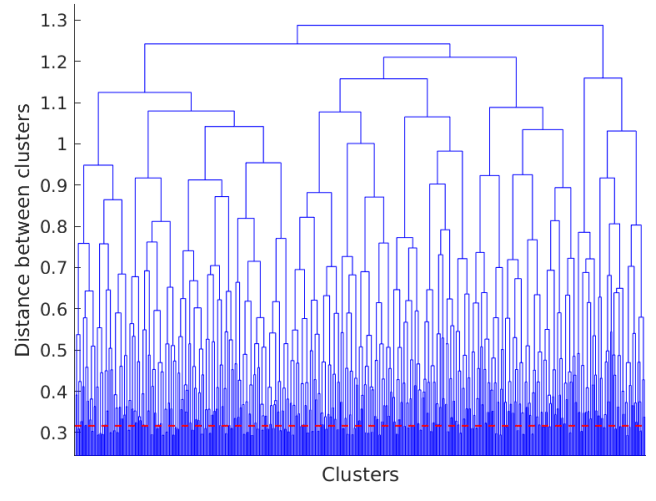

Fig. 1. Example of dendrogram for the 32 electrodes MI montage. The red horizontal line indicates the threshold $T$ for cluster separation

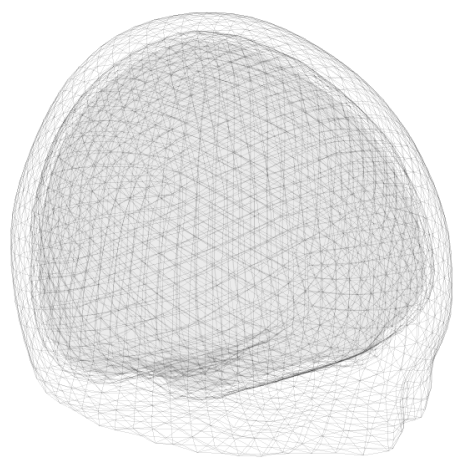

Fig. 2. 3-layer head model (Colin27)

is based on the correlation: if two scalp maps (i.e. potentials on the sensors) are correlated below 0.95 , we consider them as different. As the correlation is equal to the cosine in eq. (6), the threshold on the dendrogram is readily computed as $T=\sqrt{2(1-0.95)}=0.316$.

\section{Simulation and Results}

\section{A. Simulation setup}

The absolute potentials (forward problem) were simulated as follows: a three shell mesh model (Colin 27) was extracted from Brainstorm [11] toolbox (see Fig. 2) in order to have a realistic geometry. The cortical layer mesh (inner shell) had 7292 nodes, while the scalp mesh (outer shell) had 1922 nodes.

A regularly spaced grid was constructed inside the inner shell (brain), with a $7 \mathrm{~mm}$ distance between neighbouring points, which yielded $N=6184$ points (dipole positions). For each position, three elementary dipoles were considered.

Three standard sensor setups were tested using $M=$ $\{19,32,63\}$ scalp electrodes placed on the head surface (outer shell) according to the 10-10 system. A fourth set was specially designed for Motor Imagery tasks: it contained 32 electrodes placed on the scalp, above the motor-sensory areas and the occipital areas (figure 3). Sensor coordinates were 


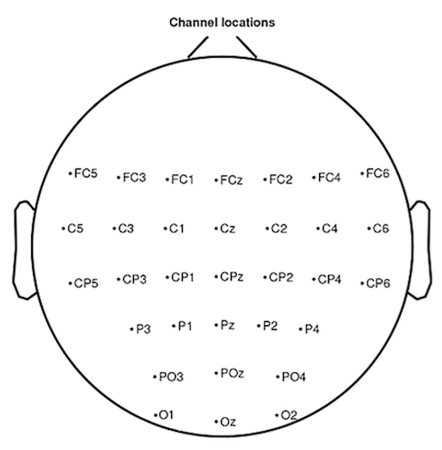

Fig. 3. Motor Imagery montage

also extracted from the Brainstorm toolbox and snapped to the mesh vertices by nearest-neighbour rule.

For each sensor setup, the absolute EEG scalp potentials $\mathbf{V}$ were simulated by projecting the sources of interest to the sensors using a lead field matrix $\mathbf{K}(M \times 3 N)$ computed using the BEM model implemented in the Helsinki toolbox [12], with conductivity ratios of $40: 1$ between the brain and the skull and 1:1 between brain and scalp.

\section{B. Results}

As expected, none of the sensor setups is able to distinguish between all $N$ subspaces. Recall that each subspace is generated by 3 lead-field columns, corresponding to dipoles situated $7 \mathrm{~mm}$ away one from another and that two subspaces are indistinguishable if their minimum correlation (cosine of the largest principal angle) is above 0.95. But the results are very different from one sensor setup to another. The number of distinguishable clusters starts by increasing with the number of sensors, as expected (the more sensors you have, the better the resolution - see table I $)^{1}$. But the most important factor influencing the resolution seems to be the spatial distribution of the sensors. Indeed, the last column of the table shows the number of clusters distinguishable using the 32 electrodes Motor Imagery montage, yielding a far lower resolution than spatially distributed montages, including the simple 10-20 montage of 19 electrodes. These

TABLE I

NUMBER OF DISTINGUISHABLE CLUSTERS DEPENDING ON THE SENSOR SETUP

\begin{tabular}{c|c|c|c||c} 
& \multicolumn{4}{|c}{ Sensor setup } \\
\hline & 19 & 32 & 63 & $32 \mathrm{MI}$ \\
\hline \# clust & 617 & 666 & 737 & 426
\end{tabular}

global results are reinforced by a more in depth analysis. As one could expect, the clusters of similar dipoles do not have the same volume. As it can be seen in fig. 4, the farther away a cluster is from the sensors, the biggest its volume. But for the well distributed montages all clusters have dimensions

\footnotetext{
${ }^{1}$ It is noteworthy that first results on a much denser setup (256, BioSemi montage) show that the resolution stops increasing. These results will be elaborated elsewhere.
}

below $10 \mathrm{~cm}^{3}$, while for the $32 \mathrm{MI}$ montage, the volumes can increase well above $20 \mathrm{~cm}^{3}$. Of course, the big clusters are situated far from the sensors, in this case in the frontal regions (see example in figure 6.

Important differences exist also among well distributed montages, as shown in figure 5. For low density montages (19 electrodes), there are almost no distinguishable regions (clusters) having volumes below $1 \mathrm{~cm}^{3}$, corresponding to 3 dipole positions (for our grid of $7 \mathrm{~mm}$, a volume covered by a dipole is roughly $0.34 \mathrm{~cm}^{3}$ ), while for high density montages one could in principle obtain much smaller distinguishable regions.

A specific question could be investigated for the $32 \mathrm{MI}$ montage: even if globally it yields a lower resolution than the classical 32 or even 19 electrode montages, one might expect to have a better resolution in the parietal and occipital areas, where the sensors are densely placed. Still, this intuition is not confirmed by the cluster number analysis limited to the dipoles situated in these specific areas. More precisely, if we focus on the dipoles situated within a given distance from the 32MI sensors, we can quantify how they are regrouped together by the clustering procedure (or equivalently, in how many distinguishable regions one can split the occipitoparietal area below the electrodes). The results are given in table II, for dipoles situated at less than $20 \mathrm{~mm}$ or at less than $30 \mathrm{~mm}$ from the $32 \mathrm{MI}$ sensors.

TABLE II

NUMBER OF DISTINCT REGIONS (CLUSTERS) OBTAINED USING DIFFERENT MONTAGES, IN THE OCCIPITO-PARIETAL AREA (DIPOLES SITUATED AT AT MOST 20 OR 30 MM FROM THE 32MI ELECTRODES)

\begin{tabular}{c|c|c} 
Montage & $<20 \mathrm{~mm}$ (118 dipoles) & $<30 \mathrm{~mm}(962$ dipoles $)$ \\
\hline 19 & 71 & 204 \\
32 & 75 & 254 \\
63 & 78 & 262 \\
$32 \mathrm{MI}$ & 73 & 234
\end{tabular}

\section{CONClusion AND Future Work}

We propose in this paper an original and quantifiable method for subsampling the lead-field matrix for source localization applications. More precisely, we introduce a novel distance between two possible dipole positions in the brain, based on subspace correlation between the corresponding lead-field groups of columns (each dipole lies in a subspace spanned by 3 lead-field columns associated to the dipole position). This distance allows us to cluster the brain in distinguishable regions (i.e., dipoles within a cluster cannot be reliably distinguished, meaning that each cluster can be represented by a single dipole).

We evaluate different EEG montages using the proposed technique. While some of the results are expected (although not yet quantified to the best of our knowledge), others are more unexpected. In particular, we have shown that the obtained resolution increases with the number of sensors, but that their spatial distribution is more important than their number. This conclusion extends also to brain regions situated below densely sampled scalp regions, like in BCI 

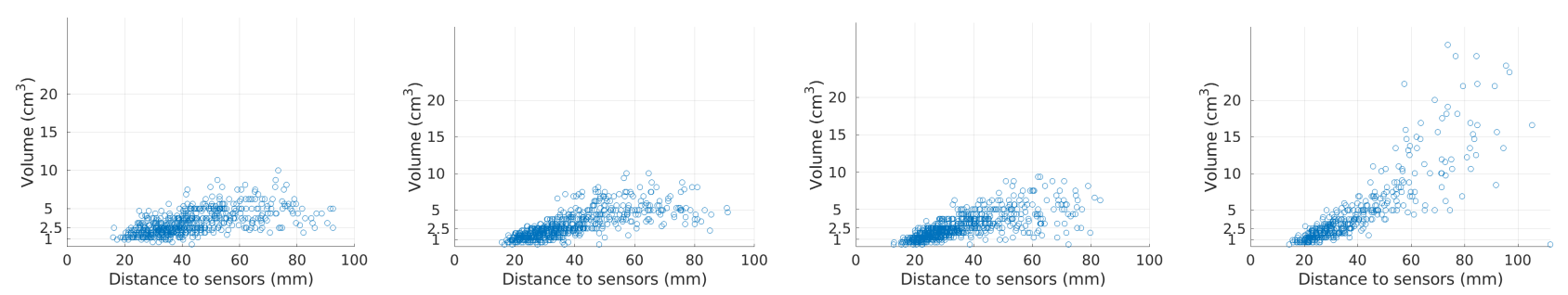

Fig. 4. Volume of the obtained clusters with respect to their distance to the sensors (the centroid of the cluster with respect to the closest sensor). From left to right, 19, 32, 63 and 32MI montages
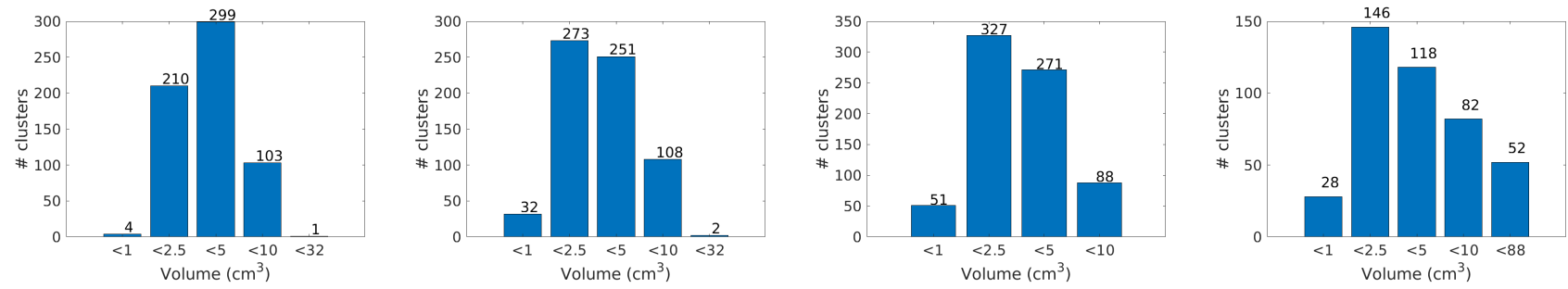

Fig. 5. Histograms presenting the number of clusters by volume, for the 4 analyzed montages. The number of clusters having volumes in the intervals $[0,1],[1,2.5],[2.55],\left[\begin{array}{ll}5 & 10\end{array}\right]$ and above are given above each bar

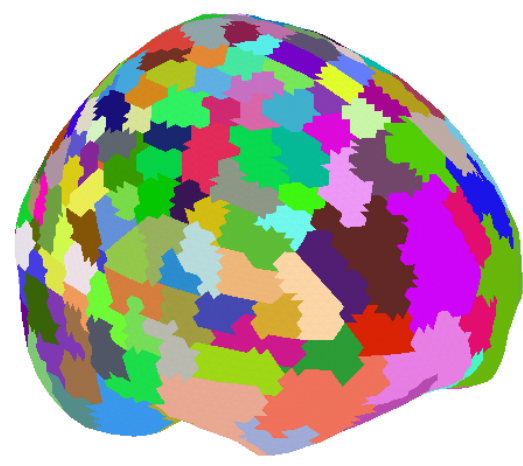

Fig. 6. Cortex parcelation yielded by the 32MI montage. Note the big cluster surfaces in the frontal ares (uncovered areas) compared to parietal areas (covered by sensors)

applications. For the Motor Imagery montage used in [13] for example, the number of separable brain regions in the areas of interest (occipito-parietal) does not benefit from the increased number of sensors above these areas.

Although more results are needed, the first conclusion seems to be that using dense but local montages does not improve source localization, and these montages are not adequate if one aims to estimate active brain regions during specific BCI tasks.

Clearly, this research needs to be extended to denser montages (128 and 256 electrodes) and confronted with atlas based segmentation of the human brain: which functional areas can be distinguished?, how many equivalent dipoles are needed for a given region?, etc. Besides, inverse problem (source localization) needs to be performed after the clustering in order to completely validate our approach.

\section{REFERENCES}

[1] M. G. Wentrup, K. Gramann, E. Wascher, and M. Buss, "Eeg source localization for brain-computer-interfaces," in Conference Proceedings. 2nd International IEEE EMBS Conference on Neural Engineering, 2005., March 2005, pp. 128-131.

[2] B. Kamousi, Z. Liu, and B. He, "Classification of motor imagery tasks for brain-computer interface applications by means of two equivalent dipoles analysis," IEEE Transactions on Neural Systems and Rehabilitation Engineering, vol. 13, no. 2, pp. 166-171, June 2005.

[3] F. Lotte, A. Lécuyer, and B. Arnaldi, "FuRIA: An inverse Solution based Feature Extraction Algorithm using Fuzzy Set Theory for Brain-Computer Interfaces," IEEE Transactions on Signal Processing, vol. 57, no. 8, pp. 3253-3263, 2009. [Online]. Available: https://hal.inria.fr/inria-00368282

[4] B. J. Edelman, B. Baxter, and B. He, "Eeg source imaging enhances the decoding of complex right-hand motor imagery tasks," IEEE Transactions on Biomedical Engineering, vol. 63, no. 1, pp. 4-14, Jan 2016.

[5] J. Song, C. Davey, C. Poulsen, P. Luu, S. Turovets, E. Anderson, K. Li, and D. Tucker, "Eeg source localization: sensor density and head surface coverage," Journal of neuroscience methods, vol. 256, pp. 9-21, 2015.

[6] L. Duque-Muñoz, F. Vargas, and J. D. López, "Simplified eeg inverse solution for bci real-time implementation," in 2016 38th Annual International Conference of the IEEE Engineering in Medicine and Biology Society (EMBC), Aug 2016, pp. 4051-4054.

[7] P. Nunez and R. Srinivasan, Electric fields of the brain: the neurophysics of EEG. Oxford University Press, USA, 2006.

[8] S. Baillet, J. Mosher, and R. Leahy, "Electromagnetic brain mapping," IEEE Signal Processing Magazine, vol. 18, no. 6, pp. 14-30, 2001.

[9] G. H. Golub and C. F. V. Loan, Matrix Computation. John Hopkins University Press, 1996

[10] R. O. Duda, P. E. Hart, and D. G. Stork, Pattern Classification (2Nd Edition). New York, NY, USA: Wiley-Interscience, 2000.

[11] S. Baillet, J. C. Mosher, R. M. Leahy, and D. W. Shattuck, "Brainstorm: a matlab toolbox for the processing of meg and eeg signals," NeuroImage, vol. 9, pp. S246-S246, 1999.

[12] M. Stenroos, V. Mäntynen, and J. Nenonen, "A matlab library for solving quasi-static volume conduction problems using the boundary element method," Computer methods and programs in biomedicine, vol. 88, no. 3, pp. 256-263, 2007.

[13] S. Rimbert, N. Gayraud, L. Bougrain, M. Clerc, and S. Fleck, "Can a subjective questionnaire used as brain-computer interface performance predictor?" submitted, 2018 